\title{
Macromolecular crowding limits growth under pressure
}

\author{
Baptiste Alric $^{1}$, Cécile Formosa-Dague ${ }^{2}$, Etienne Dague ${ }^{3}$, Liam J. Holt ${ }^{4, \dagger} \&$ Morgan Delarue $^{1, \dagger}$ \\ ${ }^{1}$ MILE team, CNRS, UPR8001, LAAS-CNRS, 7 Avenue du Colonel Roche, F-31400 Toulouse, France ${ }^{2}$ TBI, Université de Toulouse, CNRS, \\ INRAE, INSA, Toulouse, France ${ }^{3}$ ELIA team, CNRS, UPR8001, LAAS-CNRS, 7 Avenue du Colonel Roche, F-31400 Toulouse, France \\ ${ }^{4}$ New York University Grossman School of Medicine, Institute for Systems Genetics, 435 E 30th Street, New York, NY, United States \\ $\dagger$ to whom correspondence should be addressed: liam.holt@nyulangone.org; morgan.delarue@laas.fr
}

Cells that grow in confined spaces eventually build up mechanical compressive stress. This growth-induced pressure (GIP) decreases cell growth. GIP is important in a multitude of contexts from cancer, to microbial infections, to biofouling, yet our understanding of its origin and molecular consequences remains limited. Here, we combine microfluidic confinement of the yeast Saccharomyces cerevisiae, with rheological measurements using genetically encoded multimeric nanoparticles (GEMs) to reveal that growth-induced pressure is accompanied with an increase in a key cellular physical property: macromolecular crowding. We develop a fully calibrated model that predicts how increased macromolecular crowding hinders protein expression and thus diminishes cell growth. This model is sufficient to explain the coupling of growth rate to pressure without the need for specific molecular sensors or signaling cascades. As molecular crowding is similar across all domains of life, this could be a deeply conserved mechanism of biomechanical feedback that allows environmental sensing originating from the fundamental physical properties of cells.

Cells in every kingdom of life can proliferate in spatiallylimited environments. In metazoans, tissues have physical boundaries[1]. In plants, roots sprout into a solid ground[2 3]. In microbes, substrate adhesion physically limits colony expansion [4-6]. To proliferate in confinement, cells must push on the boundaries of their environment and neighboring cells, leading to development of compressive forces that translate, at the multicellular scale, into the buildup of a mechanical growthinduced pressure, hereafter denoted GIP. GIP decreases cell growth and division of all organisms: bacteria, fungi, plants or mammals[7-[12]. However, the mechanisms that control proliferation under GIP remain unknown. In particular, it is unclear whether growth reduction is due to specific signaling pathways, or is a necessary consequence to changes of the physical properties of the cells.

Some signaling pathways have been associated with survival or division under GIP[13, 14], but it remains unclear if these pathways affect growth per se. For example, mutants in the SCWISh network tend to lyse due to mechanical instabilities associated with budding, but their ability to develop GIP is unperturbed[14].
On the other hand, mechanical perturbations to cells also influence fundamental physical parameters. One such parameter is macromolecular crowding, which relates to the high packing fraction of macromolecules in the cell, and can decrease biochemical reaction rates due to decreased effective diffusion [1518]. However, the role of crowding in response to mechanical stress in general, and GIP in particular, has been largely overlooked.

In this Letter, we investigated the relationship between growth-induced pressure, macromolecular crowding and cell growth in the budding yeast $S$. cerevisiae. Our results are best explained by a model in which the rates of intracellular osmolyte production and macromolecular biogenesis are intrinsically coupled. To develop GIP, osmolytes and macromolecules are produced, while cell expansion is limited, causing the cell interior to become crowded, leading to a biophysical feedback that limits cell growth.

We used microfluidic elastic chambers as a model confining 3D environment (Fig. 1a, Methods)[19]. After filling the chamber, cells pushed against their neighbors and onto their surroundings. Cells were continually fed through microchannels to prevent nutrient depletion and enable switching of media. After 10 hours of confined growth, the elastic chamber was quite deformed, almost doubling in volume. This deformation was used to measure the amount of growth-induced pressure, GIP, developed by the cells [9, 14]. We posited that, under confinement, GIP resulted from an increase in intracellular osmotic pressure, which was balanced not only by the cell wall but also by the surrounding effective elasticity of the other cells and the PDMS chamber.

In response to osmotic stress, water loss from cell leads a decreased cell volume, which increases the concentration of intracellular osmolytes. We can subdivide osmolytes into two classes, small and large, that we operationally define by their ability to freely diffuse across the nuclear pore, a cutoff value of $\sim 3 \mathrm{~nm}$ hydrodynamic radius [20]. The concentration of small osmolytes is dominated by ions and metabolites such as glycerol, while large osmolytes are macromolecules such as proteins, ribosomes and mRNA. Changes in nuclear volume are indicative of changes in cytosolic macromolecular concentration. Under osmotic stress, there is a proportional reduction of the nuclear volume, keeping the nuclear/cytoplasmic volume 
a.

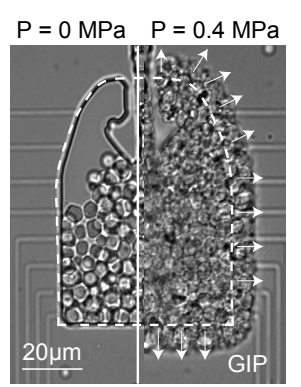

c.

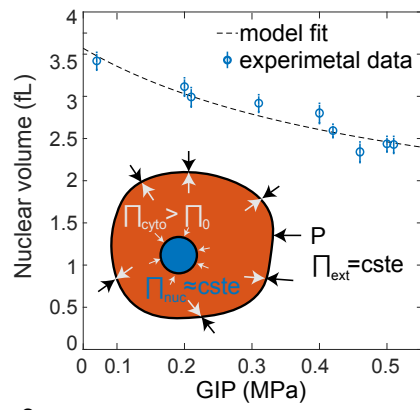

e.

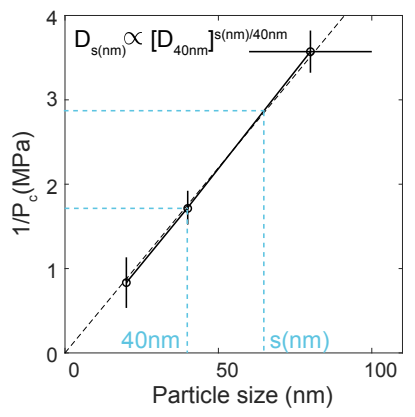

b.

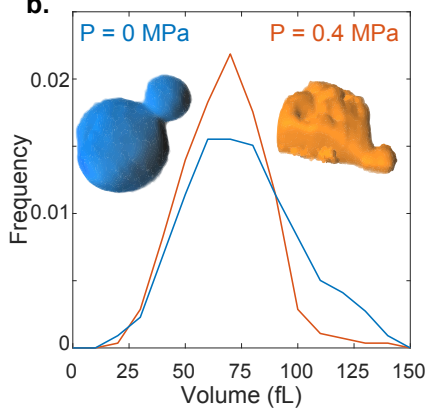

d.

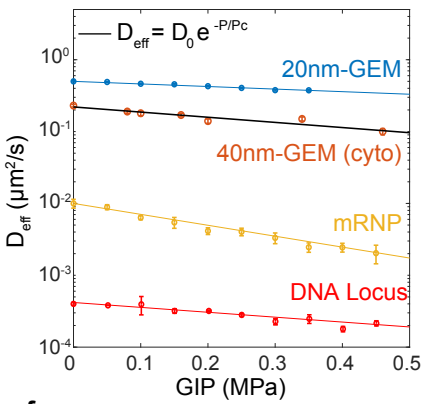

f.

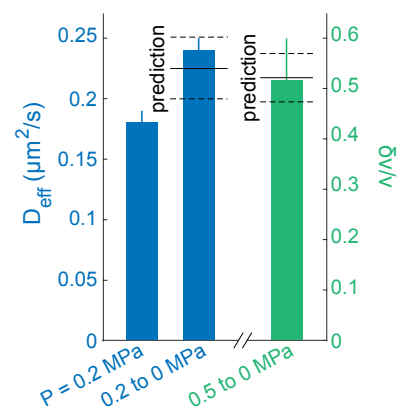

Figure 1 Confined growth leads to the intracellular accumulation of osmolytes and macromolecules. a. Confined growth leads to the build-up of growth-induced pressure (GIP), measured by the deformation of the PDMS chamber. b. Cell volume distribution under GIP. Insets: representative 3D reconstructions of a non-compressed cell and a cell at $0.4 \mathrm{MPa}$. Both cells have a similar volume $\sim 65 \mathrm{fL}$. c. Nuclear volume decreases under GIP. Dashed line: fit of nuclear volume as a function of GIP assuming constant nuclear osmotic pressure. d. Diffusivities of various particles and a DNA locus all decrease exponentially as a function of GIP. Solid black curve is the model prediction for 40nm-GEMs. e. The characteristic pressure $\left(P_{c}\right)$ of the exponential dependence is inversely proportional to cytosolic particle size. f. After sudden pressure relaxation, effective diffusion rises quickly ( $<1$ minute) to control (uncompressed) values and cell volume increases $(\delta v)$ due to stored osmotic pressure. Predicted values are indicated. In all data, values are mean \pm standard error of the mean, $\mathrm{N} \geq 3$ independent biological replicates.

ratio constant as in [21] (SI Fig. 1), indicating a relative increase in the concentration of cytoplasmic macromolecules.

Remarkably, cell size did not decrease as GIP increased (Fig. 1b), even though cells became highly deformed (Inset Fig. 1b). We posited that the deformation of cells was due to compressive forces originating from an increase in the concentration of intracellular osmolytes. Strikingly, we also observed a strong reduction in nuclear volume (Fig. 1c) and, as a result, the nuclear-to-cell volume ratio was perturbed (SI Fig. 1). Therefore, we concluded that the concentration of cytoplasmic macromolecules was also increasing. Surprisingly, our data were best fit assuming that these two osmolytes (small osmolytes and macromolecules) were increasing proportionally. Assuming that nuclear osmolarity did not adapt, we predicted that the nuclear volume $v_{n}$ would decrease with GIP, denoted $P$, as:

$$
\frac{\delta v_{n}}{v_{n}}=-\frac{P / \Pi_{0}}{1+P / \Pi_{0}}
$$

where $\Pi_{0}$ is the intracellular nominal $(\mathrm{P}=0 \mathrm{MPa})$ osmotic pressure and $P$ corresponds to the surplus internal osmotic pressure above $\Pi_{0}$. Fitting to this model yielded $\Pi_{0} \sim 0.95 \pm 0.05$ $\mathrm{MPa}$ (dashed line Fig. 1c). We measured the osmotic pressure of the culture medium at $30^{\circ} \mathrm{C}$ to be $\Pi_{e} \sim 0.63 \mathrm{MPa}$, leading to a nominal osmotic pressure difference between the cell interior and the cell exterior $\Delta \Pi \sim 0.3 \mathrm{MPa}$, in agreement with values from the literature[22]. Since macromolecule concentration was increasing while cell volume remained constant, we predicted that macromolecular crowding would increase under GIP.

Changes in macromolecular crowding can be inferred by particle tracking microrheology[23]. We recently developed genetically-encoded multimeric (GEM) nanoparticles as highly efficient tracer particles for microrheology [23]. Introduction of a gene that encodes a self-assembling scaffold protein tagged with a fluorescent protein generates cells that constitutively contain tracer particles of defined sizes. In this study, we used GEMs of $20 \mathrm{~nm}$ (20nm-GEMs) and $40 \mathrm{~nm}$ (40-nm-GEMs) diameter. These particles probe the mesoscale, the length-scale of multimeric macromolecular assemblies such as RNA polymerase and ribosomes.

Using probes of various sizes, we found that the increase in cytoplasmic crowding under mechanical compression depended strongly on length-scale (Fig. 1d): the effective diffusion of larger particles such as mRNA ( $\sim 80 \mathrm{~nm}$ diameter[24]), decreased far more than that of smaller particles such as $20 \mathrm{~nm}$ GEMs. We also found that the diffusion of a DNA locus decreased with GIP, probably as a consequence of decreased nuclear volume leading to increased nuclear crowding. Interestingly, the diffusivity of every tracer particle followed a similar exponential trend:

$$
D=D_{0} e^{-P / P_{c}}
$$


a.

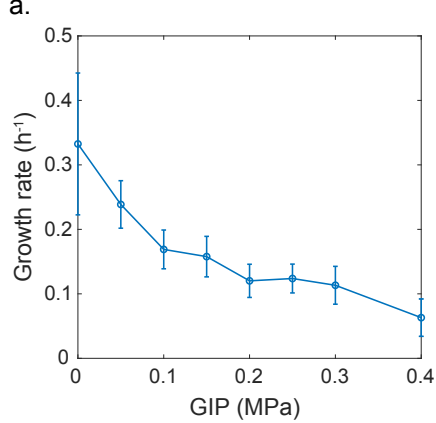

C.

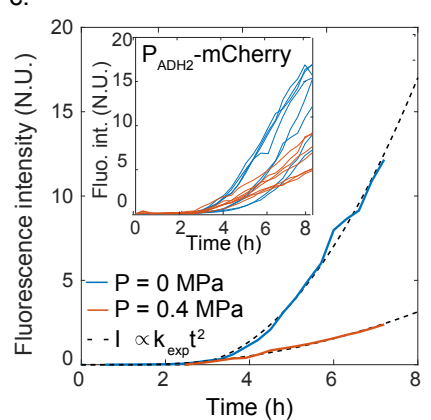

b.

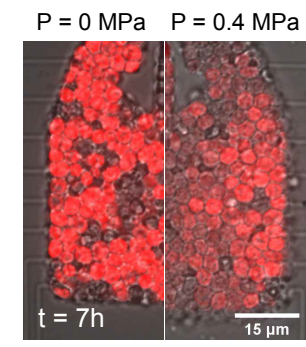

Glucose $\rightarrow \mathrm{P}_{\mathrm{ADH} 2}-$ mCherry

d.

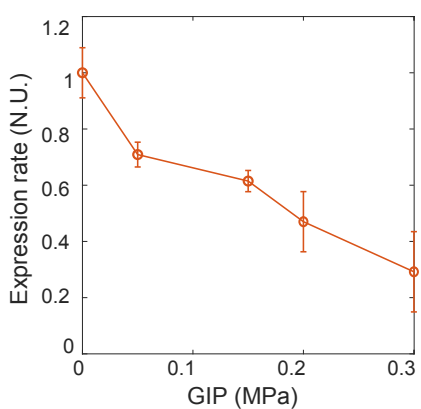

Figure 2 Confinement decreases growth and protein production rates. a. Growth rate decays roughly exponentially with GIP. b. Representative images from protein production reporter system. A reporter gene consisting of the mCherry fluorescent protein under the control of the $A D H 2$ promoter was integrated at the endogenous locus; glucose starvation induces the gene. After $7 \mathrm{~h}$ of induction, we observe stronger induction for control (no pressure, left) condition than under GIP (right). c. Single cell fluorescence intensities were fit with a quadratic function to extract an effective expression rate $k_{\exp }$ at various values of GIP. Representative curves for a single cell is shown with fitting; multiple single cell traces are shown inset. d. Protein expression rate decreases roughly exponentially with GIP. In all data, values are mean \pm standard error of the mean, over $n \geq 100$ cells in $\mathrm{N} \geq 3$ independent biological replicates.

Where $D_{0}$ is the nominal diffusion of each particle, $P$ is the growth induced pressure (GIP), and $P_{c}$ is the characteristic pressure of the exponential dependence on GIP for each particle. This exponential dependence of diffusion on GIP is theoretically predicted from the Doolittle relationship, as previously described[23] (see Modeling in SI). However, as above, this prediction only applies if osmolytes and macromolecules maintain a fixed, proportional concentration. We found that $P_{c} \propto \Pi_{i} / \zeta$, where $\zeta$ is a constant related to the interactions of the nanoparticle with its surroundings. Using osmotic perturbations to instantaneously modify crowding (SI Fig. 2), we were able to measure $P_{c} \sim 0.6 \mathrm{MPa}$ for $40 \mathrm{~nm}-\mathrm{GEMs}$. Using this value our theory predicts well our empirical data (solid black line in Fig. 1d is the prediction, red dots are the data).

Experimentally, we found that the characteristic pressure $P_{c}$ depended on particle size, and was inversely proportional to the probe size $s\left(1 / P_{c}=\beta s\right.$ where $\beta$ is the proportionality constant, Fig. 1e). This inverse relation implies that the effective cytosolic diffusion for a particle of any size, $s$ (in $\mathrm{nm}$ ) is a power law function of the diffusion at $40 \mathrm{~nm}: D_{s} \propto e^{-\beta s P}=e^{-\beta s P * 40 / 40}=$ $\left(e^{-\beta * 40 * P}\right)^{s / 40} \propto D_{40 \mathrm{~nm}}{ }^{s / 40}$. Using this relationship, we can predict cytosolic diffusivity at any length-scale from the effective diffusion of $40 \mathrm{~nm}-\mathrm{GEMs}\left(D_{40 \mathrm{~nm}}\right)$.

Our data show that confined growth leads to a concomitant increase in both internal osmotic pressure (leading to GIP) and macromolecular crowding (as evidenced by nuclear compaction and decreased nanoparticle diffusivity). Theory successfully predicts these observations if the increase in GIP and crowding are proportional. Another prediction of this proportional coupling is that relaxation of mechanical stress should lead to a cell volume increase proportional to GIP (see Modeling), $\delta v / v=P / \Pi_{0}$; and for macromolecular crowding (and thus diffusivity) to reset to the nominal value without GIP. To test this prediction, we used a device in which GIP could be quickly relaxed. Consistent with our model, we observed a fast, fully reversible and predictable increase in cell volume, and recovery of GEM diffusion upon instantaneous relaxation of GIP (Fig. 1f). Thus, confined growth leads to a proportional increase in osmolyte and macromolecule concentration.

We next sought to investigate how GIP affects cell growth and protein production (which is dependent on the rates of multiple biochemical reactions). We first measured changes in cell number and chamber volume to estimate the cellular growth rate (Methods, SI Fig. 3). We observed that growth rate decreased roughly exponentially with GIP (Fig. 2a). To get insight into protein production, we used a fluorescent reporter assay. Protein production can take hours, raising the problem that GIP would continue to increase during the experiment if growth continued. To avoid this issue, we expressed the mCherry fluorescence protein from the $A D H 2$ promoter $\left(P_{\mathrm{ADH} 2}-m\right.$ Cherry) as our model system. The $A D H 2$ promoter is activated by glucose starvation, a condition that also arrests cell growth[25]. Thus, we could grow cells to develop a defined amount of GIP and then induce $P_{\mathrm{ADH} 2}-m$ Cherry by withdrawal of glucose (osmotically balancing with sorbitol) at a range of GIP values. In this way, we could infer how protein expression, at least of this model gene, was affected by GIP. We observed that the induction of the fluorescence signal was slower under GIP than in the control (Fig. 2b).

This experimental strategy enabled us to extract single-cell $P_{\mathrm{ADH} 2-m C h e r r y}$ fluorescence intensity curves. We observed that, after an initial time delay, which could be associated with sensing of carbon starvation or promoter remodeling, fluorescence intensity increased with time, and that this rate of increase was lower in compressed cells. We developed a simple mathematical model of transcription followed by translation to quantify induction of fluorescence. Our model predicted that 
protein concentration should increase quadratically with time, with an effective rate $k_{\exp }$ that is the product of the transcription rate $\left(k_{\mathrm{m}}\right)$ and the translation rate $\left(k_{\mathrm{p}}\right): k_{\exp }=k_{\mathrm{m}} * k_{\mathrm{p}}$ (see Modeling). Although $k_{\exp }$ was not stricto sensu a rate, but rather the product of two rates, we refer to it as a single effective rate hereafter, for the sake of simplicity.

Our simple model yielded an excellent fit to the experimental data (Fig. 2c), and enabled us to extract both the time delay and $k_{\text {exp. }}$. We observed that the time delay was progressively shorter with GIP (SI Fig. 4). It has previously been shown that cells in the G1 phase of the cell cycle respond more rapidly to stress [25], and our previous studies showed that $S$. cerevisiae arrests in G1 in response to GIP[9, 26]. Therefore, accumulation of cells in G1 could explain this reduced lag-time under GIP. We found that $k_{\exp }$ decreased roughly exponentially with GIP (Fig. 2d), with a similar dependence as the growth rate decay (about 60\% decrease at $P=0.3 \mathrm{MPa}$ in both cases).

Our microrheology data and nuclear compression demonstrated that macromolecular crowding increased under GIP. We hypothesized that this crowding could limit protein expression rate and ultimately growth itself. This feedback could be physical as a result of decreases in the rate of diffusion-limited processes, with no need for specific signaling pathways. To test this idea, we set out to perturb molecular crowding by orthogonal means, using osmotic compression. HOGl is a key kinase in the osmotic stress pathway. hog $1 \Delta$ mutant cells cannot actively regulate their osmolarity [27], allowing us to maintain osmotic compression for sufficient time to assess growth and protein expression rates. Consistent with our hypothesis, we found that $k_{\mathrm{exp}}$ of $P_{\mathrm{ADH} 2}-m C h e r r y$ decreased with osmotic compression (Fig. 3a).

GIP and osmotic compression are orthogonal means of increasing molecular crowding and activate distinct stress response pathways. Furthermore, the osmotic stress response is largely abrogated in $h o g 1 \Delta$ cells [27]. If the effective expression rate $\left(k_{\exp }\right)$ of $P_{\mathrm{ADH} 2}-m$ Cherry is modulated by macromolecular crowding, then $k_{\text {exp }}$ should display the same relationship to the effective diffusion of $40 \mathrm{~nm}$-GEMs $\left(D_{40 \mathrm{~nm}}\right)$ under both GIP and osmotic compression. Indeed, we observed the same dependence in both conditions (Fig. 3b) supporting the hypothesis that macromolecular crowding limits protein expression.

Our results are consistent with effective protein expression rate being diffusion-limited at a certain unknown length-scale, $s$. We found that the relationship between effective diffusion and particles diameter was a power law (Fig 1e). If crowding decreases $P_{\mathrm{ADH} 2}-m$ Cherry production by inhibiting diffusion of a rate-limiting particle, effective expression rate should be a power law function of $D_{40 \mathrm{~nm}}$ with an exponent that is the ratio of the particle size, $s$ (in $\mathrm{nm}$ ), divided by the size of $40 \mathrm{~nm}$ GEMs (i.e. $40 \mathrm{~nm}$ ):

$$
k_{\exp } \propto D_{s} \propto D_{40 \mathrm{~nm}} s / 40
$$
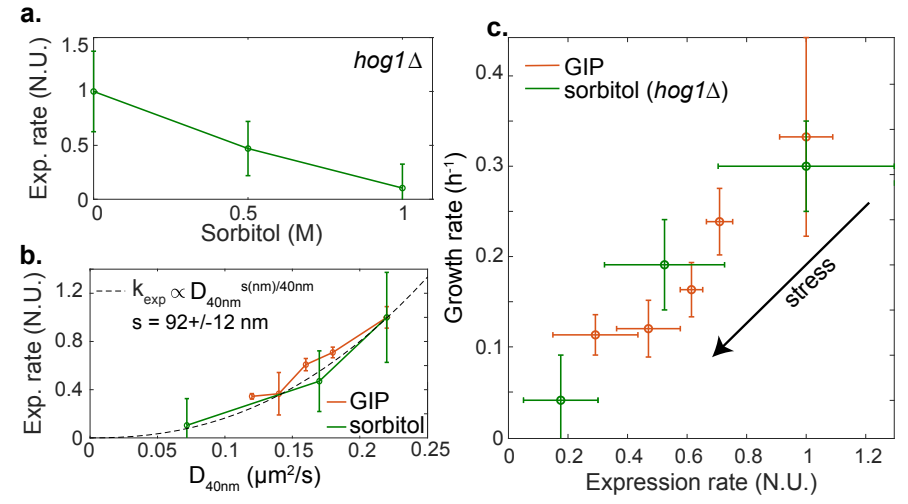

Figure 3 Protein production and growth are diffusion limited processes. a. Effective expression rate $\left(k_{\mathrm{exp}}\right)$ of $P_{\mathrm{ADH} 2}-m$ Cherry decreases under osmotic stress in $h o g 1 \Delta$ cells. b. $k_{\text {exp }}$ is a power-law function of the effective diffusion of $40 \mathrm{~nm}-\mathrm{GEMs}$. c. Growth rate is proportional to protein production rate for $h o g 1 \Delta$ cells under osmotic stress and $W T$ cells under GIP. In all data, values are mean \pm standard error of the mean, over $\mathrm{N} \geq 3$ independent biological replicates.

Indeed, we observed this power-law dependence, with an exponent that suggests that expression was limited by the diffusion of particles of a characteristic size $s \sim 90 \mathrm{~nm}$ (Fig 3b). This mesoscale length-scale corresponds to many biological entities, for example trafficking vesicles and mRNA ribonucleoprotein particles[24, 28, 29] (both $\sim 100 \mathrm{~nm}$ ).

We next investigated the hypothesis that growth rate is mainly limited by protein expression. We plotted growth rate as a function of the effective expression rate of $P_{\mathrm{ADH} 2}-m C h e r r y$ and found that the two rates were roughly proportional. Note, this model gene is not limiting growth-rate, $A D H 2$ is not expressed in the presence of glucose. Nevertheless, the fundamental processes required for its expression (e.g. transcription by RNA polymerase II and translation by ribosomes) are shared by all proteins. Consistent with the hypothesis that crowding limits both protein expression and cell growth, exactly the same relationship held for both osmotic compression of hog $1 \Delta$ cells and WT cells under GIP (Fig. 3c).

Taking all of our results together, we developed a model of confined growth, with all parameters experimentally determined, allowing us to predict protein production and cell growth in confined conditions. The model derivation and parameterization are detailed in the Supplementary Information.

Our data support a central hypothesis that osmolyte and macromolecule production rates are tightly coupled. Exactly how this balance of rates is achieved remains unknown and is a longstanding fundamental question, but as a consequence, in the absence of confinement, cells grow and accumulate biomass while maintaining a constant level of macromolecular crowding. The accumulation of osmolytes increases osmotic pressure. The mechanical balance between osmotic pressure and 
the elastic properties of the cell wall in turn defines the turgor pressure[30]. This turgor pressure enables the cell wall to expand through a process of hydrolysis and insertion of new cell wall material[31, 32]. We posit that insertion of cell wall material is only possible when the turgor pressure resulting from osmolyte accumulation is above a fixed value.

If the elasticity of the cell wall were to increase (i.e. require more force to be deformed), a higher pressure-difference, and thus more osmolytes, would be required to achieve expansion. This is also the case during confined growth where the surroundings mechanically resist cell growth. Confined growth leads to an effective increase in the elasticity around the cell which then physically limits cell wall expansion (Fig. 4a). In our experiments, when cells fill the confining chamber and start to distort one another and the chamber walls, they experience an effective surrounding elasticity, $E_{\text {eq }}$. When cells grow by $\delta v$, they need to accumulate more osmolytes to expand the cell wall, resulting in an increased internal pressure, which is the product of the surrounding elasticity and the volume change: $E_{\mathrm{eq}} * \delta v / v$. This value is the growth-induced pressure (GIP). Based on our central hypothesis that the accumulation of osmolytes is proportionally coupled to the accumulation of macromolecular biomass, the decreased expansion rate will lead to increased crowding. This increase in crowding then feeds back onto both protein and osmolyte production, which further reduces the cell expansion rate (Fig 4a).

We calibrated the parameters related to our confining growth model, including the value of the turgor pressure, using laser ablation, transmission electron microscopy and atomic force microscopy (Supplementary Information).

Our experimentally calibrated model accurately predicted the dependence of protein production and cell growth rate on pressure, as well as the dynamics of confined cell proliferation and GIP buildup, without any fitting of free parameters (thick orange lines in Figs. 4b-d). This remarkable predictive power supports our simple model: Growth is initially limited by the surrounding elastic environment, which forces the cell to increase internal osmolarity. Osmolyte production is directly coupled to macromolecular biosynthesis thus leading to mesoscale crowding. High mesoscale intracellular crowding then physically inhibits reactions through diffusion-limited processes. Our model shows that most of the observed decrease in growth rate can be explained by this physical feedback, without the need to evoke any other mechanism.

We also investigated the predictions of our model if we removed the physical feedback (thick red lines in Figs. 4bd). In this case, GIP and cell number would rise much more quickly than experimentally observed. Growth would still ultimately decrease, due to the increasing mechanical barrier to cell expansion, but much more slowly than observed because the rate of osmolyte production would not be limited. In this case, crowding would also rise quickly, and crowders in the
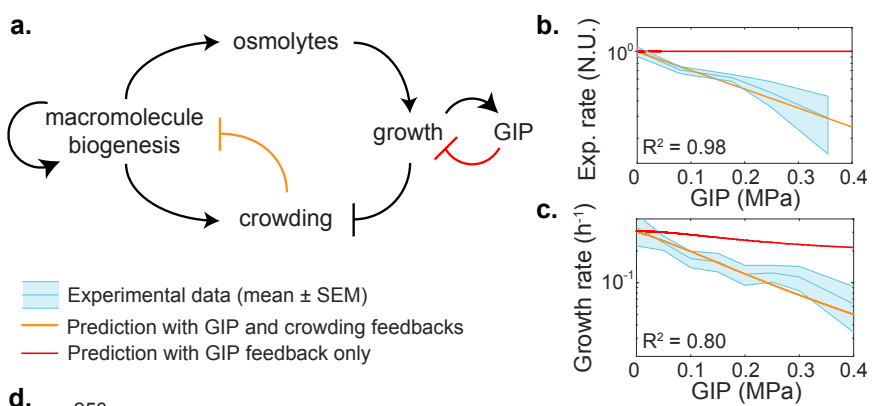

Experimental data (mean \pm SEM)

- Prediction with GIP and crowding feedbacks

- Prediction with GIP feedback only

d.
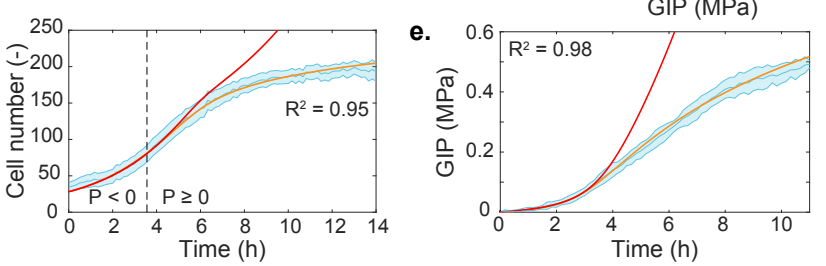

Figure 4 A physical feedback model, in which crowding limits protein production, predicts the dynamics of confined cell growth. a. Schematic of the model. After confluence is reached, growth induced pressure (GIP) increases as a function of cell volume change and the elasticity of the surrounding cells and PDMS chamber walls. Cells must accumulate more osmolytes to grow in the face of increasing effective elasticity, therefore volume expansion is inhibited. Macromolecule biogenesis is proportionally coupled to osmolyte production and so intracellular crowding increases. Increased mesoscale crowding feeds back onto many processes including the processes associated with macromolecule biogenesis itself, thereby limiting growth. b-e. Predictions of the dependence of various observables on GIP from the model. All parameters are experimentally determined. Predictions are shown for: effective protein expression rate $\left(k_{\exp }, \mathbf{b}\right)$; growth rate $(\mathbf{c})$; cell number $(\mathbf{d})$; and GIP (e). In all plots, the thick orange line represents the model prediction. The thick red line represents the prediction of the model without any physical (crowding) feedback on biomass production. The dashed line represents the onset of confluence and GIP buildup. The $R^{2}$ value indicates the square difference of the model against the data. Values are mean \pm standard error of the mean; $\mathrm{N} \geq 3$ independent biological replicates.

cell would approach the maximum random close packing fraction much sooner. We speculate that the physical feedback of crowding on biosynthesis is adaptive, as it delays and attenuates macromolecular overcrowding, which could allow more time for stress responses to more efficiently activate.

An intriguing question is why cells have not evolved adaptive mechanisms to change the relative rates of macromolecular biosynthesis and osmolyte production to prevent overcrowding of the cell. The osmotic stress response is an adaptive mechanism of this type. However, we observed that GIP in $\operatorname{hog} 1 \Delta \mathrm{mu}-$ tants, which are defective for the osmotic stress pathway, was similar to that in wild-type cells (SI Fig. 5). A key difference between GIP and osmotic shock is the effect on turgor. The activation of the osmoadaptive HOGl pathway in S. cerevisiae is 
linked with a loss of turgor[33]. However, our results suggest that turgor does not decrease during GIP, and in fact increases due to the elasticity of the surroundings effectively acting like a thicker cell wall. Increased turgor actually triggers the hypoosmotic stress response, which decreases intracellular osmolarity and subsequently cell volume[34]. However, this would be counterproductive during confined growth as reduced cell volume would further increase crowding. Indeed, pathways related to the response to both hyper- and hypo-osmotic stress are triggered by GIP. These pathways, which together constitute the SCWISh network [14], are important for cell survival under GIP, but they do not appear to change the coupling between osmolyte and macromolecule biosynthesis. Perhaps the feedback between mesoscale crowding and growth is useful: diffusion is affected with a strong size-dependence, mainly limiting reactions at the mesoscale ( $\geq 10 \mathrm{~nm}$ diameter). It is intriguing that many stress response proteins are relatively small. Therefore, upon developing strong growth-induced pressure, growth will stall, but stress-response pathways can continue to operate.

Stress-response signaling pathways vary extensively between organisms. In contrast, high macromolecular crowding is a fundamental property of all life forms [35]. Our results suggest that a primordial biophysical feedback mechanism arises directly from the physical properties of cells.

\section{Acknowledgments}

We thank E. Kassianidou for help with laser ablation experiments. We thank NYULH DART Microscopy Laboratory Alice Liang, Chris Petzold and Kristen Dancel-Manning for consultation and assistance with TEM work; this core is partially funded by NYU Cancer Center Support Grant NIH/NCI P30CA016087. The technological realisations and associated research works were partly supported by the French RENATECH network. LJH was funded by NIH grants R01 GM132447 and R37 CA240765. We thank E. Rojas for fruitful discussions. $\mathrm{LJH}$ and MD thank the FACE foundation for travel support.

\section{Author Contributions}

$\mathrm{BA}$ and $\mathrm{MD}$ designed and performed the experiments and data analysis; CCD and ED performed the AFM experiments; $\mathrm{LJH}$ designed the strains used in the study; BA, LJH and MD wrote the manuscript.

\section{References}

[1] Sebastian J Streichan, Christian R Hoerner, Tatjana Schneidt, Daniela Holzer, and Lars Hufnagel. Spatial constraints control cell proliferation in tissues. Proceedings of the National Academy of Sciences, 111(15):55865591, 2014.

[2] AG Bengough, C Croser, and J Pritchard. A biophysical analysis of root growth under mechanical stress. In Plant Roots-From Cells to Systems, pages 107-116. Springer, 1997.

[3] Kateryna Fal, Niklas Korsbo, Juan Alonso-Serra, Jose Teles, Mengying Liu, Yassin Refahi, Marie-Edith Chabouté, Henrik Jönsson, and Olivier Hamant. Tissue folding at the organ-meristem boundary results in nuclear compression and chromatin compaction. Proceedings of the National Academy of Sciences, 118(8), 2021.

[4] Natsuko Rivera-Yoshida, Juan A Arias Del Angel, and Mariana Benítez. Microbial multicellular development: mechanical forces in action. Current opinion in genetics $\&$ development, 51:37-45, 2018.

[5] Munehiro Asally, Mark Kittisopikul, Pau Rué, Yingjie $\mathrm{Du}$, Zhenxing $\mathrm{Hu}$, Tolga Çağatay, Andra B Robinson, Hongbing Lu, Jordi Garcia-Ojalvo, and Gürol M Süel. Localized cell death focuses mechanical forces during 3d patterning in a biofilm. Proceedings of the National Academy of Sciences, 109(46):18891-18896, 2012.

[6] Miguel Trejo, Carine Douarche, Virginie Bailleux, Christophe Poulard, Sandrine Mariot, Christophe Regeard, and Eric Raspaud. Elasticity and wrinkled morphology of bacillus subtilis pellicles. Proceedings of the National Academy of Sciences, 110(6):2011-2016, 2013.

[7] Philip S Stewart and Channing R Robertson. Microbial growth in a fixed volume: studies with entrapped escherichia coli. Applied microbiology and biotechnology, 30(1):34-40, 1989.

[8] Eric K Chu, Onur Kilic, Hojung Cho, Alex Groisman, and Andre Levchenko. Self-induced mechanical stress can trigger biofilm formation in uropathogenic escherichia coli. Nature communications, 9(1):1-10, 2018.

[9] Morgan Delarue, Jörn Hartung, Carl Schreck, Pawel Gniewek, Lucy Hu, Stephan Herminghaus, and Oskar Hallatschek. Self-driven jamming in growing microbial populations. Nature physics, 12(8):762-766, 2016.

[10] Gabriel Helmlinger, Paolo A Netti, Hera C Lichtenbeld, Robert J Melder, and Rakesh K Jain. Solid stress inhibits the growth of multicellular tumor spheroids. Nature biotechnology, 15(8):778-783, 1997. 
[11] Kévin Alessandri, Bibhu Ranjan Sarangi, Vasily Valériévitch Gurchenkov, Bidisha Sinha, Tobias Reinhold Kießling, Luc Fetler, Felix Rico, Simon Scheuring, Christophe Lamaze, Anthony Simon, et al. Cellular capsules as a tool for multicellular spheroid production and for investigating the mechanics of tumor progression in vitro. Proceedings of the National Academy of Sciences, 110(37):14843-14848, 2013.

[12] Ilaria Francesca Rizzuti, Pietro Mascheroni, Silvia Arcucci, Zacchari Ben-Mériem, Audrey Prunet, Catherine Barentin, Charlotte Rivière, Hélène Delanoë-Ayari, Haralampos Hatzikirou, Julie Guillermet-Guibert, et al. Mechanical control of cell proliferation increases resistance to chemotherapeutic agents. Physical Review Letters, 125(12):128103, 2020.

[13] Sungmin Nam, Vivek Kumar Gupta, Hong-pyo Lee, Joanna Y Lee, Katrina M Wisdom, Sushama Varma, Eliott Marie Flaum, Ciara Davis, Robert B West, and Ovijit Chaudhuri. Cell cycle progression in confining microenvironments is regulated by a growth-responsive trpv4-pi3k/akt-p27kip1 signaling axis. Science advances, 5(8):eaaw6171, 2019.

[14] Morgan Delarue, Gregory Poterewicz, Ori Hoxha, Jessica Choi, Wonjung Yoo, Jona Kayser, Liam Holt, and Oskar Hallatschek. Scwish network is essential for survival under mechanical pressure. Proceedings of the National Academy of Sciences, 114(51):13465-13470, 2017.

[15] Agnès Miermont, François Waharte, Shiqiong $\mathrm{Hu}$, Megan Nicole McClean, Samuel Bottani, Sébastien Léon, and Pascal Hersen. Severe osmotic compression triggers a slowdown of intracellular signaling, which can be explained by molecular crowding. Proceedings of the National Academy of Sciences, 110(14):5725-5730, 2013.

[16] Mahesh A Vibhute, Mark H Schaap, Roel JM Maas, Frank HT Nelissen, Evan Spruijt, Hans A Heus, Maike MK Hansen, and Wilhelm TS Huck. Transcription and translation in cytomimetic protocells perform most efficiently at distinct macromolecular crowding conditions. ACS Synthetic Biology, 2020.

[17] Guilherme Nettesheim, Ibtissem Nabti, Chandrashekhar U Murade, Gabriel R Jaffe, Stephen J King, and George T Shubeita. Macromolecular crowding acts as a physical regulator of intracellular transport. Nature Physics, pages 1-8, 2020.

[18] Jorge Fernandez-de Cossio-Diaz and Alexei Vazquez. A physical model of cell metabolism. Scientific reports, 8(1):1-13, 2018.
[19] Liam J Holt, O Hallatschek, and Morgan Delarue. Mechano-chemostats to study the effects of compressive stress on yeast. Methods in cell biology, 147:215-231, 2018.

[20] Dirk Görlich and Ulrike Kutay. Transport between the cell nucleus and the cytoplasm. Annual review of cell and developmental biology, 15(1):607-660, 1999.

[21] Elzbieta Petelenz-Kurdziel, Emma Eriksson, Maria Smedh, Caroline Beck, Stefan Hohmann, and Mattias Goksör. Quantification of cell volume changes upon hyperosmotic stress in saccharomyces cerevisiae. Integrative Biology, 3(11):1120-1126, 2011.

[22] IM De Mara on, Pierre-André Marechal, and Patrick Gervais. Passive response of saccharomyces cerevisiae to osmotic shifts: cell volume variations depending on the physiological state. Biochemical and biophysical research communications, 227:519-523, 1996.

[23] Morgan Delarue, Gregory P Brittingham, Stefan Pfeffer, IV Surovtsev, S Pinglay, KJ Kennedy, M Schaffer, JI Gutierrez, D Sang, G Poterewicz, et al. mtorc1 controls phase separation and the biophysical properties of the cytoplasm by tuning crowding. Cell, 174(2):338-349, 2018.

[24] Alexander Borodavka, Surendra W Singaram, Peter G Stockley, William M Gelbart, Avinoam Ben-Shaul, and Roman Tuma. Sizes of long rna molecules are determined by the branching patterns of their secondary structures. Biophysical journal, 111(10):2077-2085, 2016.

[25] Stefano Busti, Paola Coccetti, Lilia Alberghina, and Marco Vanoni. Glucose signaling-mediated coordination of cell growth and cell cycle in saccharomyces cerevisiae. Sensors, 10(6):6195-6240, 2010.

[26] Morgan Delarue, Daniel Weissman, and Oskar Hallatschek. A simple molecular mechanism explains multiple patterns of cell-size regulation. PloS one, 12(8), 2017.

[27] Markus J Tamás and Stefan Hohmann. The osmotic stress response of saccharomyces cerevisiae. Yeast stress responses, pages 121-200, 2003.

[28] Elias M Puchner, Jessica M Walter, Robert Kasper, Bo Huang, and Wendell A Lim. Counting molecules in single organelles with superresolution microscopy allows tracking of the endosome maturation trajectory. Proceedings of the National Academy of Sciences, 110(40):16015-16020, 2013.

[29] Shigeo Takamori, Matthew Holt, Katinka Stenius, Edward A Lemke, Mads Grønborg, Dietmar Riedel, Henning Urlaub, Stephan Schenck, Britta Brügger, Philippe 
bioRxiv preprint doi: https://doi.org/10.1101/2021.06.04.446859; this version posted June 4, 2021. The copyright holder for this preprint (which was not certified by peer review) is the author/funder, who has granted bioRxiv a license to display the preprint in perpetuity. It is made available under aCC-BY-NC-ND 4.0 International license.

Ringler, et al. Molecular anatomy of a trafficking organelle. Cell, 127(4):831-846, 2006.

[30] Dominic Vella, Amin Ajdari, Ashkan Vaziri, and Arezki Boudaoud. The indentation of pressurized elastic shells: from polymeric capsules to yeast cells. Journal of the Royal Society Interface, 9(68):448-455, 2012.

[31] Enrique R Rojas, Kerwyn Casey Huang, and Julie A Theriot. Homeostatic cell growth is accomplished mechanically through membrane tension inhibition of cell-wall synthesis. Cell systems, 5(6):578-590, 2017.

[32] Peter Albersheim, Alan Darvill, Keith Roberts, Ron Sederoff, and Andrew Staehelin. Plant cell walls. Garland Science, 2010.

[33] Jörg Schaber, Miquel Angel Adrover, Emma Eriksson, Serge Pelet, Elzbieta Petelenz-Kurdziel, Dagmara Klein, Francesc Posas, Mattias Goksör, Mathias Peter, Stefan Hohmann, et al. Biophysical properties of saccharomyces cerevisiae and their relationship with hog pathway activation. European Biophysics Journal, 39(11):1547-1556, 2010 .

[34] David E Levin. Cell wall integrity signaling in saccharomyces cerevisiae. Microbiology and molecular biology reviews, 69(2):262, 2005.

[35] R John Ellis. Macromolecular crowding: obvious but underappreciated. Trends in biochemical sciences, 26(10):597-604, 2001. 